\title{
Prevalence of PALB2 Germline Mutations in Early-onset and Familial Breast/Ovarian Cancer Patients from Pakistan
}

\author{
Muhammad Usman Rashid, MBBS, PhD',2 \\ Faiz Ali Khan, MPhil ${ }^{1}$ \\ Noor Muhammad, MPhil ${ }^{1}$ \\ Asif Loya, MBBS, MD ${ }^{3}$ \\ Ute Hamann, $\mathrm{PhD}^{2}$
}

\begin{abstract}
${ }^{1}$ Department of Basic Sciences Research, Shaukat Khanum Memorial Cancer Hospital and Research Centre (SKMCHERC), Lahore, Pakistan, ${ }^{2}$ Molecular Genetics of Breast Cancer, German Cancer Research Center (DKFZ), Heidelberg, Germany, ${ }^{3}$ Department of Pathology, Shaukat Khanum Memorial Cancer Hospital and Research Centre (SKMCHERC), Lahore, Pakistan
\end{abstract}

Correspondence: Muhammad Usman Rashid, MBBS, PhD

Department of Basic Sciences Research,

Shaukat Khanum Memorial Cancer Hospital and Research Centre (SKMCH\&RC), 7A, Block R3, Johar Town, Lahore, Punjab 54000, Pakistan Tel: 92-423-5905000

Fax: 92-423-5945206

E-mail: usmanr@skm.org.pk

Received June 14, 2018

Accepted October 10, 2018

Published Online October 11, 2018

\begin{abstract}
Purpose
Partner and localizer of BRCA2 (PALB2) is a breast cancer susceptibility gene that plays an important role in DNA repair. This is the first study assessing the prevalence of PALB2 mutations in early-onset and familial breast/ovarian cancer patients from Pakistan.
\end{abstract}

\section{Materials and Methods}

PALB2 mutation screening was performed in 370 Pakistani patients with early-onset and familial breast/ovarian cancer, who were negative for BRCA1, BRCA2, TP53, CHEK2, and RAD51C mutations, using denaturing high-performance liquid chromatography analysis. Mutations were confirmed by DNA sequencing. Novel PALB2 alterations were analyzed for their potential effect on protein function or splicing using various in silico prediction tools. Three-hundred and seventy-two healthy controls were screened for the presence of the identified (potentially) functional mutations.

\section{Results}

A novel nonsense mutation, p.Y743*, was identified in one familial breast cancer patient $(1 / 127,0.8 \%)$. Besides, four in silico-predicted potentially functional mutations including three missense mutations and one $5^{\prime}$ untranslated region mutation were identified: p.D498Y, novel p.G644R, novel p.E744K, and novel c.-134_-133delTCinsGGGT. The mutations p.Y743* and p.D498Y were identified in two familial patients diagnosed with unilateral or synchronous bilateral breast cancer at the ages of 29 and 39, respectively. The other mutations were identified in an early-onset ( $\leq 30$ years of age) breast cancer patient each. All five mutations were absent in 372 healthy controls suggesting that they are disease associated.

\section{Conclusion}

Our findings show that PALB2 mutations account for a small proportion of early-onset and hereditary breast/ovarian cancer cases in Pakistan.

\section{Introduction}

Breast cancer has a substantial impact on the overall tumor burden in Pakistan comprising $40 \%$ of all female malignancies. In Pakistan, monoallelic germline mutations in the highand moderate-penetrance breast cancer susceptibility genes BRCA1, BRCA2, TP53, CHEK2, and RAD51C account for approximately $25 \%$ of early-onset and familial breast cancer suggesting that other susceptibility gene(s) may be involved.
Key words

Familial breast cancer, PALB2, Germ-line mutation, Pakistan
Recently identified PALB2 gene, a partner and localizer of BRCA2, acts as a link between BRCA2 and BRCA1 and enables DNA repair [1]. Biallelic mutations in PALB2 (FAN$C N)$ cause Fanconi anemia, with clinical features similar to those caused by biallelic mutations in BRCA2 (FANCD1) [2]. Monoallelic mutations in PALB2 confer susceptibility to breast cancer suggesting that $P A L B 2$ is another candidate to be a breast cancer susceptibility gene [3]. Deleterious PALB2 mutations are estimated to confer a $35 \%$ lifetime risk of breast cancer for the carriers [4]. 
PALB2 mutation screening in BRCA1/2-negative earlyonset or familial breast cancer patients performed previously in different populations have yielded variable results. In a British study, monoallelic mutations in PALB2 were identified in $1.1 \%$ of familial breast cancer patients $(10 / 923)$ and were absent in controls $(0 / 1,084)$ [3]. In a study conducted in Finland, a recurrent PALB2 mutation, c.1592delT, was identified in $2.7 \%$ of familial breast cancer patients $(3 / 113)$ and in $0.2 \%$ of controls $(6 / 2,501)$ [5]. Mutations were identified in families from Europe, North America, and Australia at frequencies ranging from $0.3 \%$ to $5.5 \%$ [6-9], while mutations were absent in families from Canada [10], United States [11], and Chile [12].

In Asia, little is known about the contribution of PALB2 mutations to early-onset and familial breast/ ovarian cancer. Deleterious PALB2 mutations were reported in families from China, Korea, and Malaysia/Singapore with frequencies varying from $0.8 \%$ to $1.6 \%$ [13-15] and were absent in Japanese families [16]. Given the limited data on genetic variability of PALB2 in South Asia and the fact that only $25 \%$ of hereditary breast/ovarian cancer in Pakistan is attributed to germline mutations in BRCA1/2, TP53, CHEK2, and RAD51C [17-20], we assessed and report the prevalence of PALB2 mutations in 370 early-onset and familial breast/ ovarian cancer patients from this population, who had tested negative for mutations in these five breast cancer susceptibility genes. Functional and potentially functional mutations were screened in 372 healthy controls.

\section{Materials and Methods}

\section{Study population}

The study included 370 early-onset and familial breast/ ovarian cancer patients who were diagnosed with invasive breast cancer or epithelial ovarian cancer. The patients were evaluated and accrued to the study at the Shaukat Khanum Memorial Cancer Hospital and Research Center (SKMCH\& RC) in Lahore, Pakistan, from June 2001 to January 2012. Patients were classified into six groups based on the age at the time of disease onset or family history of breast/ ovarian cancer: A1, families with one female breast cancer diagnosed $\leq 30$ years of age; A2, families with two first- or seconddegree (through a male) female relatives diagnosed with breast cancer, at least one diagnosed $\leq 50$ years of age; A3, families with at least three cases of breast cancer, at least one diagnosed $\leq 50$ years of age; A4, families with one male breast cancer case diagnosed at any age; B, families with at least one female breast cancer and one ovarian cancer at any age, and $\mathrm{C} 1$, families with at least one ovarian cancer diagnosed $\leq 45$ years of age. All index patients were previously tested and shown to be negative for deleterious mutations in the BRCA1/2, CHEK2, and RAD51C genes $[17,19,20]$. Of these index patients, 290 cases were also tested negative for disease-causative mutations in the TP53 gene [18]. Patients with bilateral breast cancer or breast and ovarian cancer were considered as two independent primary cancers. A description of the study participants is shown in Table 1.

Immunohistochemical analyses of estrogen receptor (ER),

Table 1. Frequency of PALB2 mutations according to family structure and TNBC subtype

\begin{tabular}{|c|c|c|c|}
\hline Risk group & Phenotype of families & Families $^{a)}$ & PALB2 mutation \\
\hline - & All families & 370 & $1(0.3)$ \\
\hline- & Female breast cancer families & 307 & $1(0.3)$ \\
\hline A1 & 1 case $\leq 30$ years & 180 & 0 \\
\hline $\mathrm{A} 2+\mathrm{A} 3$ & $\geq 2$ cases, $\geq 1$ diagnosed $\leq 50$ years & 127 & $1(0.8)$ \\
\hline A4 & Male breast cancer families & & \\
\hline- & $\geq 1$ case of male breast cancer & 18 & 0 \\
\hline B & Breast-ovarian cancer families & & \\
\hline- & $\geq 1$ breast cancer and $\geq 1$ ovarian cancer & 23 & 0 \\
\hline- & Ovarian cancer families & & \\
\hline $\mathrm{C} 1$ & $\geq 1$ case $(\mathrm{s}), \geq 1$ diagnosed $\leq 45$ years & 22 & 0 \\
\hline Subtype & - & & \\
\hline TNBC & - & 101 & $1(1.0)$ \\
\hline Non-TNBC & - & 217 & 0 \\
\hline Unknown & - & 24 & 0 \\
\hline
\end{tabular}

Values are presented as number (\%). TNBC, triple negative breast cancer. ${ }^{\text {a) }}$ All families were negative for $B R C A 1 / 2, C H E K 2$, and RAD51C germline mutations; 290 families were negative for TP53 germline mutations. 
progesterone receptor (PR), and human epidermal growth factor receptor 2 (HER2) expression were performed on the breast tumor of the index patients as described previously [21].

The control group comprised of 372 healthy Pakistani women. Their recruitment details have been previously reported [22].

\section{Molecular analyses}

Genomic DNA extraction was performed as described previously [22]. The complete coding sequence and exon-intron junctions of the PALB2 gene (GenBank accession number NM_024675.3) were screened in the 370 index patients by denaturing high-performance liquid chromatography (DHPLC) analysis using WAVE 4500 DNA Fragment Analysis System (Transgenomics, Omaha, NE). Custom designed polymerase chain reaction (PCR)-primer pairs were used (Transgenomic Personalized Customer Support). DHPLC is a temperature modulated heteroduplex analysis which relies upon the physical changes in DNA molecules induced by mismatch heteroduplex formation [23]. Heteroduplexes were formed by denaturing the PCR product. The amplified product was loaded on a unique DNA separation matrix (Transgenomics) with homoduplexes and heteroduplexes eluting to it differentially under denaturing conditions. The elution profiles of heterduplexes were easily distinguished from those of homoduplexes. This technique has also been reported previously to detect PALB2 mutations [11,13,24,25]. When available, a mutation positive control for each exon was included in each analysis. Primer sequences, the setup of PCR reactions, cycling conditions, and DHPLC running conditions are available upon request.

Samples revealing variant DHPLC profiles were bi-directionally sequenced using an automated 3500 Genetic Analyzer (Applied Biosystems, Foster City, CA). The presence of identified pathogenic and in silico-predicted potentially deleterious PALB2 mutations was assessed in 372 controls.

\section{In silico analyses}

All identified novel PALB2 missense variants were analyzed for their potential effect on protein function by using five different computational algorithms, Align-GVGD, SIFT, MutationTaster, Polyphen-2, and SNAP2 in the default settings. In brief, Align-GVGD is based on the biophysical features of amino acids and multiple sequence alignments of protein (http:// agvgd.iarc.fr/agvgdinput.php). SIFT predicts the evolutionary conservation of amino acids within protein families (http://sift.jcvi.org). MutationTaster prediction is based on regulatory features such as histone modification sites or transcription factor binding sites, evolutionary con- servation of amino acid or nucleotide, and splice site predictions (http://www.mutationtaster.org/). PolyPhen-2 predicts the influence of an amino acid substitution on the structure and function of human protein (http:// genetics.bwh. harvard.edu/pph2/). SNAP2 is based on multiple sequence alignment and predict changes on the secondary structure and compare the solvent accessibility for mutant and wild type proteins (http://www.rostlab.org/services/snap/submit) [26].

Novel intronic variants were also analyzed for their potential effect on splicing using the splice prediction algorithms, SpliceSiteFinder-like (http:// www.umd.be/ searchSpliceSite. html), MaxEntScan (http://genes.mit.edu/burgelab/maxent/), NNSPLICE (http://www.fruitfly.org/seq_tools/splice. html), GeneSplicer (http://ccb.jhu.edu/software/genesplicer/), and HumanSplice Finder (http://www.umd.be /HSF/) by means of the Alamut software interface (Interactive Biosoftware) in default settings. All these algorithms compare the splice site signal of wild type sequence with that of the mutated sequence. Moreover, one novel variant identified in $5^{\prime}$ untranslated region (5' UTR) of PALB2 was analyzed to predict its potential effect on transcription factor binding sites using PROMO (v3.0.2) on-line program (http:// alggen. lsi.upc.es/cgibin/promo_v3/promo/promoinit.cgi?dirDB= TF_8.3).

\section{Ethical statement}

Informed written consent was obtained from all study participants prior to providing a blood sample. The study was approved by the Institutional Review Board (IRB) of the SKMCH\&RC (IRB approval number ONC-BRCA-001/2).

\section{Results}

The present study included 370 early-onset and familial breast/ovarian cancer patients from Pakistan, who were negative for BRCA1/2, TP53, CHEK2, and RAD51C mutations. Of these, 180 were diagnosed with early-onset breast cancer ( $\leq 30$ years of age), 127 belonged to families with two or more breast cancers, 23 to families with both breast and ovarian cancer, 22 to families with at least one ovarian cancer (diagnosed $\leq 45$ years of age), and 18 to families with male breast cancer (Table 1). The median age of the disease onset was 30 years (range, 19 to 73 years) for female breast cancer $(n=324)$, 47.5 years (range, 30 to 73 years) for male breast cancer $(\mathrm{n}=18)$, and 33 years (range, 22 to 60 years) for ovarian cancer $(n=35)$. One-hundred and one index patients presented with triple-negative breast cancer (TNBC) and 217 with non- 


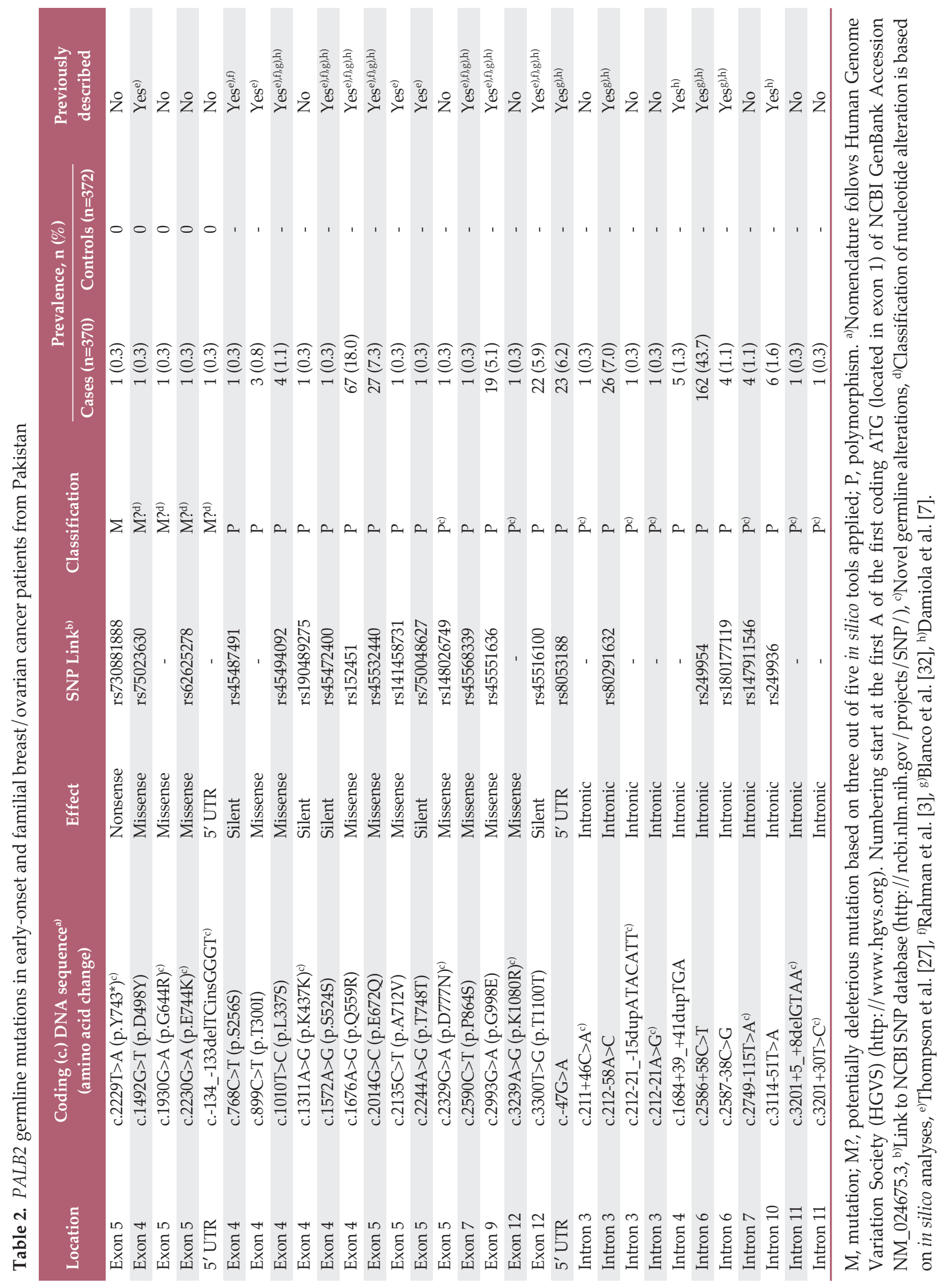


Table 3. In silico analyses of PALB2 coding variants identified in early-onset and familial breast/ovarian cancer patients from Pakistan

\begin{tabular}{|c|c|c|c|c|c|c|}
\hline \multirow{2}{*}{ Coding variant } & \multicolumn{5}{|c|}{ In silico prediction tool } & \multirow{2}{*}{ Consensus $^{a}$} \\
\hline & PolyPhen-2 & SIFT & Align GVGD & MutationTaster & SNAP2 & \\
\hline c.1492G>T (p.D498Y)b) & Possibly damaging & Deleterious & $\mathrm{CO}$ & Polymorphism & Effect & Deleterious (3/5) \\
\hline c.1930G>A (p.G644R) & Benign & Deleterious & $\mathrm{C} 15$ & Polymorphism & Effect & Deleterious $(3 / 5)$ \\
\hline c.2230G >A (p.E744K) & Possibly damaging & Deleterious & $\mathrm{CO}$ & Polymorphism & Effect & Deleterious (3/5) \\
\hline c.2329G >A (p.D777N) & Benign & Tolerated & $\mathrm{CO}$ & Polymorphism & Neutral & Benign \\
\hline c.3239A >G (p.K1080R) & Probably damaging & Tolerated & $\mathrm{C} 0$ & Polymorphism & Neutral & Benign \\
\hline
\end{tabular}

PolyPhen-2, Polymorphism Phenotyping ver. 2; SIFT, Sorting Intolerant From Tolerant; Align GVGD, Alignment of

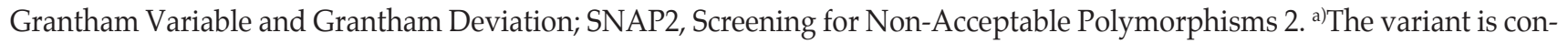
sidered as deleterious by three of the five protein function algorithms, ${ }^{b}$ Previously reported missense variant $[14,16,27]$.

Table 4. In silico analyses of PALB2 noncoding variants identified in early-onset and familial breast/ovarian cancer patients from Pakistan

\begin{tabular}{|c|c|c|c|c|c|c|}
\hline \multirow[b]{2}{*}{ Noncoding variant } & \multicolumn{5}{|c|}{ Splice-site predictions } & \multirow[b]{2}{*}{ Consensus $^{\mathrm{a}, \mathrm{b})}$} \\
\hline & SpliceSiteFinder-like & MaxEntScan & NNSPLICE & GeneSplicer & $\begin{array}{l}\text { HumanSplice } \\
\text { Site Finder }\end{array}$ & \\
\hline $\begin{array}{l}\text { c.-134_- } \\
\text {-133delTCinsGGGT }\end{array}$ & $\mathrm{NE}$ & $\mathrm{D}(0$ to 6.2$)$ & $\mathrm{NE}$ & $\mathrm{D}(0$ to 4.1$)$ & $\mathrm{D}(0$ to 82.1$)$ & Deleterious $(3 / 5)$ \\
\hline c. $211+46 \mathrm{C}>\mathrm{A}$ & $\mathrm{NE}$ & A (8.6 to 6.8$)$ & A (1.0 to 0.7$)$ & $\mathrm{NE}$ & $\mathrm{NE}$ & Benign \\
\hline $\begin{array}{l}\text { c.212-21_ } \\
\text {-15dupATACATT }\end{array}$ & NE & NE & $\mathrm{NE}$ & $\mathrm{NE}$ & NE & Benign \\
\hline c. $212-21 \mathrm{~A}>\mathrm{G}$ & NE & NE & NE & NE & NE & Benign \\
\hline c. $2749-115 \mathrm{~T}>\mathrm{A}$ & NE & $\mathrm{A}(4.7$ to 2.7$)$ & NE & NE & NE & Benign \\
\hline с. $3201+5$ +8delGTAA & $\mathrm{NE}$ & D (11.0 to 5.5$)$ & NE & $\mathrm{D}(4.43$ to 1.1$)$ & NE & Benign \\
\hline c. $3201+30 \mathrm{~T}>\mathrm{C}$ & NE & NE & NE & NE & NE & Benign \\
\hline
\end{tabular}

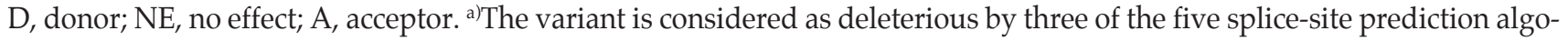
rithms, b) $>20 \%$ change in score (i.e., a wild-type splice-site score decreases, and/or a cryptic splice-site score increases) is considered as significant.

TNBC. The median age of the disease onset was 28 years (range, 19 to 67 years) for patients with TNBC and 30 years (range, 19 to 73 years) for those with non-TNBC.

Overall, 31 different heterozygous PALB2 variants were detected. Of these, 13 were novel: one nonsense mutation, four missense variants, one silent variant, six intronic variants, and one 5' UTR variant (Table 2). The remaining 18 variants have been previously reported in other populations: eight missense variants, four silent variants, five intronic variants, and one $5^{\prime}$ UTR variant.

The novel nonsense mutation at nucleotide position 2229 in exon 5, c.2229T>A (p.Y743*), was detected in a 29-year-old breast cancer patient of Punjabi ethnicity. The patient presented with a grade 3 , invasive ductal carcinoma (IDC) of TNBC phenotype. A sister of the index patient was diag- nosed with breast cancer at the age of 41 years.

The remaining 12 novel variants were analyzed for their potential functional effect by in silico analysis. Two missense variants p.G644R and p.E744K and the previously reported missense variant p.D498Y $[14,16,27]$ were predicted to be potentially deleterious by three out of five in silico analysis tools (Table 3). The 5' UTR variant c.-134_-133-del-TCinsGGGT was predicted to create a splice donor site by three out of five splice-site prediction tools implying that it was disease associated (Table 4). This variant was also predicted to create the binding sites for two transcription factors, GR-alpha (T00337) and RXR-alpha (T01345), using PROMO (v3.0.2) on-line program.

The potentially disease-causative missense mutations p.G644R and p.E744K were identified in two women of Pun- 
jabi or Pathan ethnic group, who were diagnosed with earlyonset breast cancer at the ages of 30 years, respectively. Their tumors were grade 3 IDCs, which were, ER-positive, PR-positive, and HER2-negative. The other missense mutation, p.D498Y, was identified in a patient of Pathan origin, who was diagnosed with synchronous bilateral breast cancer at 39 years of age. Left-sided breast tumor was grade 2 IDC and ER-positive, PR-negative, and HER2-negative. Right-sided breast tumor was grade 3 IDC and ER-negative, PR-negative, and HER2-positive. The index patient reported a family history of breast cancer and other cancers. The mother and one sister of the index patient were diagnosed with breast cancer at the ages of 53 and 44 years, respectively. Her father was diagnosed with lung cancer at age 69 . One sister and paternal uncle of the index patient were diagnosed to have brain cancer at the ages of 34 and 48 years, respectively.

The 5' UTR variant, c.-134_-133delTCinsGGGT, was identified in an early-onset breast cancer patient of Pathan ethnic group, who was diagnosed with disease at 26 years of age. Her tumor was a grade 2 IDC of TNBC phenotype. This patient had also been diagnosed with adenocarcinoma of the rectum at 25 years of age.

The nonsense mutation and the four in silico-predicted potentially functional mutations were not identified in 372 controls implying that they were disease-causative (Table 2). The remaining 17 variants (seven missense variants, four silent variants, five intronic variants, and one 5 ' UTR variant) were reported previously and classified as polymorphisms (Table 2).

\section{Discussion}

In this large study conducted in Pakistan, we assessed for the first time the prevalence of $P A L B 2$ germline mutations in 370 early-onset and familial breast/ovarian cancer patients negative for BRCA1/2, TP53, CHEK2, and RAD51C mutations. One novel deleterious and four in silico-predicted potentially functional PALB2 mutations (including three novels) were identified. Our study provides additional data on the contribution of PALB2 mutations to hereditary breast/ ovarian cancer in an Asian population from Pakistan.

The novel pathogenic PALB2 mutation (p.Y743*) was identified in $0.8 \%$ of families with two or more breast cancers (1/127). Similar mutation frequencies were reported in other Asian breast and / or ovarian cancer families from Saudi Arabia $(5 / 725,0.7 \%)$ [28], China $(3 / 360,0.8 \%)$ [13], and Korea $(2 / 235,0.8 \%)$ [14]. Higher frequencies were reported in studies conducted in India $(9 / 752,1.2 \%)$ [29], Malaysia/Singapore $(2 / 122,1.6 \%)$ [15], China $(2 / 108,1.8 \%)$ [30], and Korea
$(3 / 120,2.5 \%)$ [31]. This could be due to the Next-Generation Sequencing approach employed in most of these studies. Furthermore, our results are likely to underestimate the PALB2 mutation frequencies as the recently reported large genomic rearrangements were not screened [29,32]. Mutations were not identified in few studies from Japan [16], Chile [12], and United States [11]. In European and North American studies including more than 100 families from Czech Republic, France, Ireland, Germany, Poland, Italy, Finland, Canada, United States, and Australia the mutation frequencies ranged from $0.3 \%$ to $5.5 \%$ [5-9,33-38]. Altogether these findings suggest that PALB2 mutations account for a small proportion of hereditary breast/ ovarian cancer in most populations including the Pakistani population.

Four in silico-predicted potentially functional PALB2 mutations (p.G644R, p.E744K, p.D498Y, and c.-134_-133delTCinsGGGT) were also identified in this study. The novel missense mutations p.G644R and p.E744K, each identified in an early-onset breast cancer patient, were located in the highly conserved MRG15-binding domain of PALB2 spanning amino acid residues 611 to 764 . They may ablate PALB2 interacting with MRG15, which may result in impaired DNA repair [39] leading to genomic instability and cancer. The p.G644R variant was predicted to be deleterious by SIFT, Align-GVGD and SNAP-2 algorithms, while it was predicted as benign by PolyPhen-2 and MutationTaster. The differential prediction may be due to the underlying algorithm's differences as SIFT uses evolutionary sequence conservation whereas PolyPhen-2 utilizes protein structure information [40]. Since none of these algorithms is $100 \%$ predictive, a consensus prediction is reported to improve the prediction performance [41]. Overall, this variant was considered as deleterious by three of the five protein function algorithms. The missense mutation p.D498Y was identified in a familial breast cancer patient. Due to lack of DNA samples, co-segregation of the mutation with breast cancer could not be studied. Previously, this was also reported as an in silico-predicted potentially disease-causative mutation in families from Korea, Japan, and Australia $[14,16,27]$. The 5' UTR variant, c.-134_-133delTCinsGGGT, was detected in a patient with personal history of breast and rectal cancer. It was predicted to result in activation of a cryptic splice site. This variant was also predicted to affect two transcription factor binding sites and may alter the promoter activity of PALB2. None of these mutations were detected in 372 controls further suggesting that they are disease-causative; however, functional analyses of the potentially disease-causative mutations will be required for final mutation classification.

No deleterious PALB2 mutations were identified in earlyonset breast cancer patients, families with breast and ovarian cancer, ovarian cancer, or male breast cancer. These findings are in keeping with those previously reported in families 
from Turkey [42], Finland [43], Japan [16], the Netherlands [44], Spain [32], Italy [45], and Australia [46]. In contrast, PALB2 mutations have also been reported in families from Poland [37], Italy [47], Czech Republic [8], and China [13]. These findings suggest that PALB2 mutations may not substantially confer susceptibility to early-onset breast cancer patients and patients from breast and ovarian cancer, male breast cancer or ovarian cancer families.

In our study, the breast tumors associated with the PALB2 truncating (p.Y743*) and in silico-predicted potentially functional (p.D498Y, p.G644R, and p.E744K) mutations presented with high-grade tumors of IDC histology, which is consistent with previous studies conducted among Asian [13-15], European [24,25,48], and North-American patients [49]. In the Pakistani study, the breast tumor linked with the truncating mutation displayed the TNBC phenotype, in agreement with other studies from Europe, North-America and Australia [50-52]. Breast tumors associated with missense mutations were ER-positive, PR-positive, and HER2-negative. Similar hormone receptor expression patterns were also reported in breast tumors of Asian [14], European [48], and North-American patients [53], who harbored PALB2 truncating mutations. The differential expression of hormone receptors could be due to mutation-specific tumor phenotypes in the studied population.

In summary, we have identified one novel pathogenic and four potentially pathogenic PALB2 mutations, three being novel in 370 Pakistani early-onset and familial breast/ovarian cancer patients, negative for mutations in BRCA1/2, TP53, CHEK2, and RAD51C. The frequency of PALB2 mutations in patients with familial breast cancer was $0.8 \%(1 / 127)$, while no mutations were identified in early-onset breast cancer patients and patients from breast and ovarian cancer, male breast cancer or ovarian cancer families. Our findings suggest a marginal contribution of PALB2 mutations to breast cancer susceptibility in Pakistan.

\section{Conflicts of Interest}

Conflict of interest relevant to this article was not reported.

\section{Acknowledgments}

We thank the patients and healthy individuals for their participation in this study. We are grateful to Saima Faisal for the recruitment of study participants. We thank Zdenek Kleibl, Melissa C. Southey, and Natalia Bogdanova for providing DNA samples of PALB2 mutation controls and Asim Amin for critical review of the manuscript. This study was funded by the internal fund from Shaukat Khanum Memorial Cancer Hospital and Research Centre, Lahore, Pakistan (grant number ONC-BRCA-001/2).

\section{References}

1. Xia B, Sheng Q, Nakanishi K, Ohashi A, Wu J, Christ N, et al. Control of BRCA2 cellular and clinical functions by a nuclear partner, PALB2. Mol Cell. 2006;22:719-29.

2. Howlett NG, Taniguchi T, Olson S, Cox B, Waisfisz Q, De DieSmulders C, et al. Biallelic inactivation of BRCA2 in Fanconi anemia. Science. 2002;297:606-9.

3. Rahman N, Seal S, Thompson D, Kelly P, Renwick A, Elliott A, et al. PALB2, which encodes a BRCA2-interacting protein, is a breast cancer susceptibility gene. Nat Genet. 2007;39: 165-7.

4. Antoniou AC, Casadei S, Heikkinen T, Barrowdale D, Pylkas $\mathrm{K}$, Roberts J, et al. Breast-cancer risk in families with mutations in PALB2. N Engl J Med. 2014;371:497-506.

5. Erkko H, Xia B, Nikkila J, Schleutker J, Syrjakoski K, Mannermaa A, et al. A recurrent mutation in PALB2 in Finnish cancer families. Nature. 2007;446:316-9.

6. Casadei S, Norquist BM, Walsh T, Stray S, Mandell JB, Lee $\mathrm{MK}$, et al. Contribution of inherited mutations in the BRCA2interacting protein PALB2 to familial breast cancer. Cancer Res. 2011;71:2222-9.

7. Damiola F, Schultz I, Barjhoux L, Sornin V, Dondon MG, Eon-
Marchais S, et al. Mutation analysis of PALB2 gene in French breast cancer families. Breast Cancer Res Treat. 2015;154:46371.

8. Janatova M, Kleibl Z, Stribrna J, Panczak A, Vesela K, Zimovjanova $\mathrm{M}$, et al. The PALB2 gene is a strong candidate for clinical testing in BRCA1- and BRCA2-negative hereditary breast cancer. Cancer Epidemiol Biomarkers Prev. 2013;22:2323-32.

9. Teo ZL, Park DJ, Provenzano E, Chatfield CA, Odefrey FA, Nguyen-Dumont T, et al. Prevalence of PALB2 mutations in Australasian multiple-case breast cancer families. Breast Cancer Res. 2013;15:R17.

10. Guenard F, Pedneault CS, Ouellette G, Labrie Y, Simard J; INHERIT, et al. Evaluation of the contribution of the three breast cancer susceptibility genes CHEK2, STK11, and PALB2 in non-BRCA1/ 2 French Canadian families with high risk of breast cancer. Genet Test Mol Biomarkers. 2010;14:515-26.

11. Ding YC, Steele L, Chu LH, Kelley K, Davis H, John EM, et al. Germline mutations in PALB2 in African-American breast cancer cases. Breast Cancer Res Treat. 2011;126:227-30.

12. Leyton $Y$, Gonzalez-Hormazabal P, Blanco R, Bravo T, Fernandez-Ramires R, Morales S, et al. Association of PALB2 
sequence variants with the risk of familial and early-onset breast cancer in a South-American population. BMC Cancer. 2015;15:30.

13. Cao AY, Huang J, Hu Z, Li WF, Ma ZL, Tang LL, et al. The prevalence of PALB2 germline mutations in BRCA1/BRCA2 negative Chinese women with early onset breast cancer or affected relatives. Breast Cancer Res Treat. 2009;114:457-62.

14. Kim H, Cho DY, Choi DH, Oh M, Shin I, Park W, et al. Frequency of pathogenic germline mutation in CHEK2, PALB2, MRE11, and RAD50 in patients at high risk for hereditary breast cancer. Breast Cancer Res Treat. 2017;161:95-102.

15. Phuah SY, Lee SY, Kang P, Kang IN, Yoon SY, Thong MK, et al. Prevalence of PALB2 mutations in breast cancer patients in multi-ethnic Asian population in Malaysia and Singapore. PLoS One. 2013;8:e73638.

16. Nakagomi H, Sakamoto I, Hirotsu Y, Amemiya K, Mochiduki H, Omata M. Analysis of PALB2 mutations in 155 Japanese patients with breast and/or ovarian cancer. Int J Clin Oncol. 2016;21:270-5.

17. Rashid MU, Zaidi A, Torres D, Sultan F, Benner A, Naqvi B, et al. Prevalence of BRCA1 and BRCA2 mutations in Pakistani breast and ovarian cancer patients. Int J Cancer. 2006;119: 2832-9.

18. Rashid MU, Gull S, Asghar K, Muhammad N, Amin A, Hamann U. Prevalence of TP53 germ line mutations in young Pakistani breast cancer patients. Fam Cancer. 2012;11:307-11.

19. Rashid MU, Muhammad N, Faisal S, Amin A, Hamann U. Constitutional CHEK2 mutations are infrequent in early-onset and familial breast/ovarian cancer patients from Pakistan. BMC Cancer. 2013;13:312.

20. Rashid MU, Muhammad N, Faisal S, Amin A, Hamann U. Deleterious RAD51C germline mutations rarely predispose to breast and ovarian cancer in Pakistan. Breast Cancer Res Treat. 2014;145:775-84.

21. Rashid MU, Muhammad N, Bajwa S, Faisal S, Tahseen M, Bermejo JL, et al. High prevalence and predominance of BRCA1 germline mutations in Pakistani triple-negative breast cancer patients. BMC Cancer. 2016;16:673.

22. Rashid MU, Muzaffar M, Khan FA, Kabisch M, Muhammad N, Faiz S, et al. Association between the BsmI polymorphism in the vitamin D receptor gene and breast cancer risk: results from a Pakistani case-control study. PLoS One. 2015;10: e0141562.

23. Underhill PA, Jin L, Lin AA, Mehdi SQ, Jenkins T, Vollrath D, et al. Detection of numerous $Y$ chromosome biallelic polymorphisms by denaturing high-performance liquid chromatography. Genome Res. 1997;7:996-1005.

24. Dansonka-Mieszkowska A, Kluska A, Moes J, Dabrowska M, Nowakowska D, Niwinska A, et al. A novel germline PALB2 deletion in Polish breast and ovarian cancer patients. BMC Med Genet. 2010;11:20.

25. Garcia MJ, Fernandez V, Osorio A, Barroso A, Llort G, Lazaro C, et al. Analysis of FANCB and FANCN/PALB2 fanconi anemia genes in BRCA1/2-negative Spanish breast cancer families. Breast Cancer Res Treat. 2009;113:545-51.

26. Ernst C, Hahnen E, Engel C, Nothnagel M, Weber J, Schmut- zler RK, et al. Performance of in silico prediction tools for the classification of rare BRCA1/2 missense variants in clinical diagnostics. BMC Med Genomics. 2018;11:35.

27. Thompson ER, Gorringe KL, Rowley SM, Wong-Brown MW, McInerny S, Li N, et al. Prevalence of PALB2 mutations in Australian familial breast cancer cases and controls. Breast Cancer Res. 2015;17:111.

28. Siraj AK, Masoodi T, Bu R, Parvathareddy SK, Al-Badawi IA, Al-Sanea N, et al. Expanding the spectrum of germline variants in cancer. Hum Genet. 2017;136:1431-44.

29. Singh J, Thota N, Singh S, Padhi S, Mohan P, Deshwal S, et al. Screening of over 1000 Indian patients with breast and/or ovarian cancer with a multi-gene panel: prevalence of BRCA1/ 2 and non-BRCA mutations. Breast Cancer Res Treat. 2018;170:189-96.

30. Jian W, Shao K, Qin Q, Song S, Wang X. Clinical and genetic characterization of hereditary breast cancer in a Chinese population. Hered Cancer Clin Pract. 2017;15:19.

31. Park JS, Lee ST, Nam EJ, Han JW, Lee JY, Kim J, et al. Variants of cancer susceptibility genes in Korean BRCA1/2 mutationnegative patients with high risk for hereditary breast cancer. BMC Cancer. 2018;18:83.

32. Blanco A, de la Hoya M, Balmana J, Ramon y Cajal T, Teule A, Miramar MD, et al. Detection of a large rearrangement in PALB2 in Spanish breast cancer families with male breast cancer. Breast Cancer Res Treat. 2012;132:307-15.

33. Aloraifi F, McDevitt T, Martiniano R, McGreevy J, McLaughlin $\mathrm{R}$, Egan CM, et al. Detection of novel germline mutations for breast cancer in non-BRCA1 / 2 families. FEBS J. 2015;282:342437.

34. Bogdanova N, Sokolenko AP, Iyevleva AG, Abysheva SN, Blaut M, Bremer M, et al. PALB2 mutations in German and Russian patients with bilateral breast cancer. Breast Cancer Res Treat. 2011;126:545-50.

35. Catucci I, Peterlongo P, Ciceri S, Colombo M, Pasquini G, Barile $\mathrm{M}$, et al. PALB2 sequencing in Italian familial breast cancer cases reveals a high-risk mutation recurrent in the province of Bergamo. Genet Med. 2014;16:688-94.

36. Hartley T, Cavallone L, Sabbaghian N, Silva-Smith R, Hamel $\mathrm{N}$, Aleynikova O, et al. Mutation analysis of PALB2 in BRCA1 and BRCA2-negative breast and/or ovarian cancer families from Eastern Ontario, Canada. Hered Cancer Clin Pract. 2014; 12:19.

37. Kluska A, Balabas A, Piatkowska M, Czarny K, Paczkowska $\mathrm{K}$, Nowakowska D, et al. PALB2 mutations in BRCA1/2mutation negative breast and ovarian cancer patients from Poland. BMC Med Genomics. 2017;10:14.

38. Tedaldi G, Tebaldi M, Zampiga V, Danesi R, Arcangeli V, Ravegnani $\mathrm{M}$, et al. Multiple-gene panel analysis in a case series of 255 women with hereditary breast and ovarian cancer. Oncotarget. 2017;8:47064-75.

39. Sy SM, Huen MS, Chen J. MRG15 is a novel PALB2-interacting factor involved in homologous recombination. J Biol Chem. 2009;284:21127-31.

40. Karchin R. Next generation tools for the annotation of human SNPs. Brief Bioinform. 2009;10:35-52. 
41. Chan PA, Duraisamy S, Miller PJ, Newell JA, McBride C, Bond $\mathrm{JP}$, et al. Interpreting missense variants: comparing computational methods in human disease genes CDKN2A, MLH1, MSH2, MECP2, and tyrosinase (TYR). Hum Mutat. 2007;28: 683-93.

42. Cecener G, Guney Eskiler G, Egeli U, Tunca B, Alemdar A, Gokgoz S, et al. Association of PALB2 sequence variants with the risk of early-onset breast cancer in patients from Turkey. Mol Biol Rep. 2016;43:1273-84.

43. Kuusisto KM, Bebel A, Vihinen M, Schleutker J, Sallinen SL. Screening for BRCA1, BRCA2, CHEK2, PALB2, BRIP1, RAD50, and CDH1 mutations in high-risk Finnish BRCA1 /2founder mutation-negative breast and / or ovarian cancer individuals. Breast Cancer Res. 2011;13:R20.

44. Adank MA, van Mil SE, Gille JJ, Waisfisz Q, Meijers-Heijboer H. PALB2 analysis in BRCA2-like families. Breast Cancer Res Treat. 2011;127:357-62.

45. Silvestri V, Rizzolo P, Zanna I, Falchetti M, Masala G, Bianchi $S$, et al. PALB2 mutations in male breast cancer: a populationbased study in Central Italy. Breast Cancer Res Treat. 2010;122: 299-301.

46. Sauty de Chalon A, Teo Z, Park DJ, Odefrey FA; kConFab, Hopper JL, et al. Are PALB2 mutations associated with increased risk of male breast cancer? Breast Cancer Res Treat. 2010;121: 253-5.

47. Silvestri V, Zelli V, Valentini V, Rizzolo P, Navazio AS, Coppa $\mathrm{A}$, et al. Whole-exome sequencing and targeted gene sequenc- ing provide insights into the role of PALB2 as a male breast cancer susceptibility gene. Cancer. 2017;123:210-8.

48. Heikkinen T, Karkkainen H, Aaltonen K, Milne RL, Heikkila $\mathrm{P}$, Aittomaki K, et al. The breast cancer susceptibility mutation PALB2 1592delT is associated with an aggressive tumor phenotype. Clin Cancer Res. 2009;15:3214-22.

49. Tischkowitz M, Xia B. PALB2/FANCN: recombining cancer and Fanconi anemia. Cancer Res. 2010;70:7353-9.

50. Couch FJ, Hart SN, Sharma P, Toland AE, Wang X, Miron P, et al. Inherited mutations in 17 breast cancer susceptibility genes among a large triple-negative breast cancer cohort unselected for family history of breast cancer. J Clin Oncol. 2015;33:304-11.

51. Kraus C, Hoyer J, Vasileiou G, Wunderle M, Lux MP, Fasching $\mathrm{PA}$, et al. Gene panel sequencing in familial breast/ovarian cancer patients identifies multiple novel mutations also in genes others than BRCA1/2. Int J Cancer. 2017;140:95-102.

52. Wong-Brown MW, Avery-Kiejda KA, Bowden NA, Scott RJ. Low prevalence of germline PALB2 mutations in Australian triple-negative breast cancer. Int J Cancer. 2014;134:301-5.

53. Schoolmeester JK, Moyer AM, Goodenberger ML, Keeney GL, Carter JM, Bakkum-Gamez JN. Pathologic findings in breast, fallopian tube, and ovary specimens in non-BRCA hereditary breast and/or ovarian cancer syndromes: a study of 18 patients with deleterious germline mutations in RAD51C, BARD1, BRIP1, PALB2, MUTYH, or CHEK2. Hum Pathol. 2017;70:1426. 